\title{
A Novel Spherical Packed Bed Application on Decentralized Heat Recovery Ventilation Units
}

\author{
Alper Mete GENC ${ }^{*}$, Ziya Haktan KARADENIZ², Orhan EKREN ${ }^{3}$ and Macit TOKSOY4 \\ ${ }^{1}$ The Graduate School of Natural and Applied Sciences, Mechanical Engineering Department, Kâtip Celebi University, Izmir, Turkey \\ ${ }^{2}$ Mechanical Engineering Department, Kâtip Celebi University, Cigli, Izmir, Turkey \\ ${ }^{3}$ Ege University, Solar Energy Institute, Izmir, Turkey \\ ${ }^{4}$ ENEKO, Cigli, Izmir, Turkey
}

\begin{abstract}
Decentralized heat recovery ventilation (HRV) systems are assumed as simple solutions to obtain a healthy and comfortable indoor environment. A wall or window mounted compact version of decentralized HRV systems (mono unit) are used for small scale, mostly residential applications. A fan and a heat exchanger are the critical components of this compact system. The flow capacity of these units are down to $10 \mathrm{~m}^{3} / \mathrm{h}$, where efficiencies over $90 \%$ are commonly declared by the manufacturers. On the other hand, spherical packed beds (SPD) are widely used in the heat transfer applications such as; chemical reactors, grain driers, nuclear reactors, thermal storage in buildings and in solar thermal power plants, due to operational convenience. These systems are operated under steady flow conditions, unlike decentralized HRV systems which are designed for cyclic operation. In this study, heat recovery performance of a spherical packed bed heat exchanger for a decentralized HRV system is investigated. A one dimensional mathematical model for a SPD is obtained and an in-house computer code is developed to solve the transient heat transfer inside the packed bed under cyclic operation conditions. Well known convenient correlations were used for pressure drop calculations. A number of bed and sphere diameters were studied in a wide range. Various flow time and number of cycles were studied for the hot and cold flow to understand the SPD performance for HRV applications. This novel application also has the potential for regenerative heat recovery systems.
\end{abstract}

\section{Introduction}

Various types of packed beds have the potential to increase heat and mass transfer and are widely used in chemical reactors, nuclear reactors, heat exchangers and heat storage areas [1-3]. In addition, low cost and ease of use are also shown among other reasons [4]. The design of the packed beds depends on the heat and mass transfer and the flow and pressure drop mechanisms of the fluid to the solid body bed. These mechanisms are affected by the voidage changes of the packed bed [5]. The voidage changes for the SPDs are proportional to the diameter of the bed and the spheres used. The correct selection of the voidage will be useful to calculate the pressure drop inside a packed bed, estimate the capital and operating costs, and size the fans or pumps required to flow the fluid into the system [6]. A simple SPD is shown in Figure 1.

In the literature, there are approaches that give the variation of the voidage $(\varepsilon)$ and cylinder diameter - sphere diameter $(\mathrm{D} / \mathrm{d}) \lambda$ ratio. The experimental approaches defined by [7] and [8] are shown in equations (1) and (2), respectively.

$$
\begin{gathered}
\bar{\varepsilon}=0.375+\frac{0.34}{\lambda} \\
\bar{\varepsilon}=0.41+0.35 \exp (-0.39 \lambda)
\end{gathered}
$$

Depending on the voidage, the pressure drop can be calculated. Since the main factor that determines the power of the fan, the pressure drop must be predicted with high accuracy during the design process. For the estimation of pressure drop, it is very important to use the correct approach. As a result of the theoretical and experimental studies on this subject, many approaches have been derived $[9,10]$. In a study by [9] according to the approaches in the literature, the voidage values varied between 0.351-0.485, D/d ratio 3.23-57.33, and Reynolds number values ranged 0.0095-5463. In another study, studies have been conducted with a wider range $(0.330$ 0.882 ) of cavity rates [10]. This information contradicts other sources [11], where the clearance rate for SPDs is generally reported to be between $0.3-0.5$ in the literature.

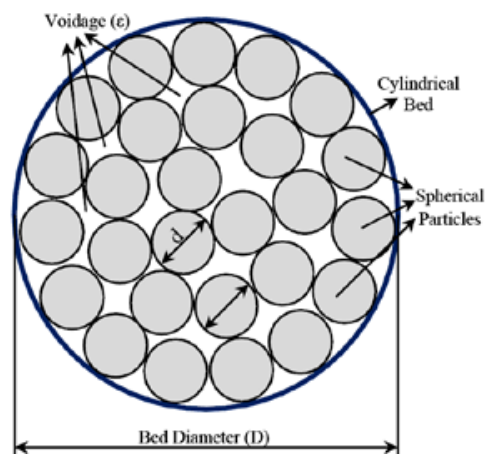

Fig.1. A Spherical packed bed (SPD)

\footnotetext{
* Corresponding author: alper.m.genc@gmail.com
} 
The most commonly used pressure drop approach for spherical packed beds is the Ergun equation [12]. Derived from experimental studies, this equation derived as an indicator of pressure losses caused by viscous and inertial energy losses. The Ergun equation is shown in Equation (3).

$$
\frac{\Delta p}{L} \frac{150(1-\epsilon)^{2}}{\epsilon^{3}} \frac{\mu_{f}}{d_{p}{ }^{2}} U 1.75 \frac{(1-\epsilon)}{\epsilon^{3}} \frac{\rho_{f}}{d_{p}} U^{2}
$$

Here, the $\Delta \mathrm{p}, \mathrm{L}, \varepsilon$ is pressure drop, bed length, the voidage, respectively. The Ergun equation developed by Mehta and Hawley (1969) [13] is shown below. Here, M physically refers to the effect of the wet surface of the wall.

$$
\begin{gathered}
\frac{\Delta p}{L}=\frac{150(1-\varepsilon)^{2}}{\varepsilon^{3}} \frac{\mu_{f} M^{2}}{d_{p}{ }^{2}} U+1.75 \frac{1-\varepsilon}{\varepsilon^{3}} \frac{\rho_{f} M}{d_{p}} U^{2} \\
M=1+\frac{2 d_{p}}{3 D(1-\varepsilon)}
\end{gathered}
$$

Experimental studies on spherical packed beds are generally related to pressure drop and voidage. However, in theoretical and numerical studies, heat and mass transfer rather than pressure drop were examined generally. Numerical methods employ as a powerful tool to investigate the fluid flow and heat transfer in packed bed due to the necessity of test equipment and long experimentation time of experimental methods.

Augier et al. (2010) [14] investigated the transport and transfer properties inside packed beds of spherical particles by means of CFD simulations at low Reynolds numbers $\left(1<\mathrm{R}_{\mathrm{e}}<100\right)$ and steady flow conditions. The properties of the system were selected as L/d: 17, D/d: 25 and $\varepsilon: 0.33-0.37$. They concluded that Nusselt number increase with particle radius depending on the effect of Reynolds number. In addition, they mentioned that Nusselt number are not dependent on the number of contacting point. Guardo et al. (2005) [15] studied the influence of the turbulence model in CFD modeling of wall-to-fluid heat transfer in packed beds. They concluded that $\mathrm{Nu}$ number and heat transfer rate increases with increasing Reynolds number.

To the best of authors' knowledge, there is no study so far on numerical transient analyses of spherical packed beds with periodic flow. Bed diameters were studied ranging between $0.18-0.2 \mathrm{~m}$. Spherical diameters were chosen in a wide range of $0.0025-0.06 \mathrm{~m}$. Various flow time and number of cycles were investigated for the hot and cold flow to understand the spherical bed performance for HRV applications. This novel application also has the potential for application on regenerative heat recovery systems.

\section{Material \& Method}

In the current work, one dimensional and transient spherical packed bed model is numerically investigated. The schematic of the problem is presented in Figure 6. For the analyses, the following assumptions have been considered;

- Thermo-physical properties of air at $20^{\circ} \mathrm{C}$ are used.

- The inlet temperatures of the air to the system are 20 and $-10^{\circ} \mathrm{C}$ for the hot and cold sides, respectively.

- The flow rate of the fluid is accepted $1 \mathrm{~m} / \mathrm{s}$.

- Thermo-physical properties of aluminum are used for spheres.

- $\quad 0.18-0.2$ diameter and $0.1 \mathrm{~m}$ length are selected for system properties. The diameter of the spheres is accepted as $0.0025-0.06 \mathrm{~m}$.

The voidage was determined to perform the analyses. Then, the pressure drop was calculated. The average convection heat transfer coefficient was calculated with the obtained voidage value and heat transfer calculation was performed.

\subsection{Pressure Drop}

As already mentioned, voidage value must be obtained accurately to calculate the pressure drop. Two different experimental approaches presented by [7] and [8] are mentioned in the literature section. The relationship between the different voidage values and the $\mathrm{D} / \mathrm{d}$ diameter ratio, which are examined according to these two approaches, are shown in Figure 1.

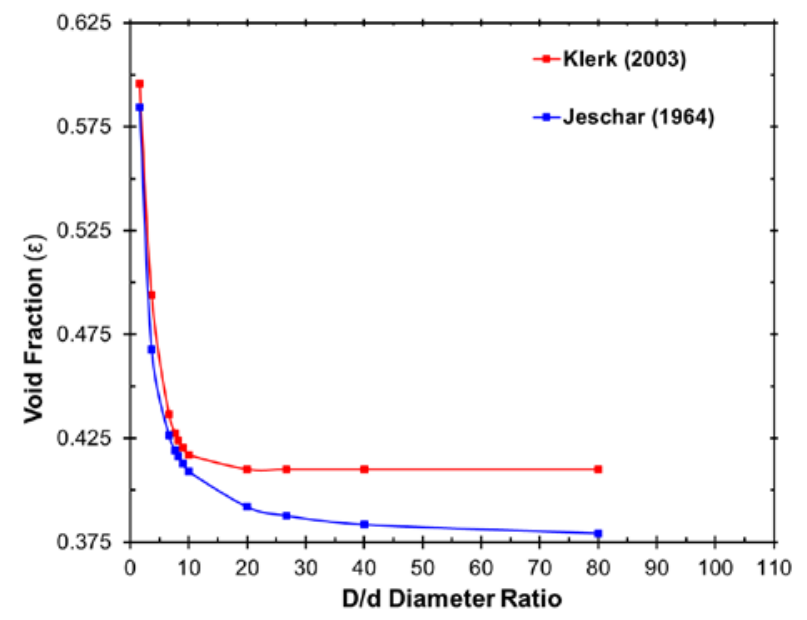

Fig. 2. Relationship between voidage and $\mathrm{D} / \mathrm{d}$ ratio

In Figure 2, it is seen that the ratio of void decreases as the $\mathrm{D} / \mathrm{d}$ ratio increases. According to the approach proposed by [8], the void ratio reaches a constant value (0.41) after a certain diameter ratio. The equation developed by [13], the thermo-physical properties of air at $20{ }^{\circ} \mathrm{C}$ and the experimentally determined maximum $\mathrm{D} / \mathrm{d}$ diameter ratio values in the literature (1.624 - 100) 
was used. The flow rate of the fluid to the heat exchanger was accepted as $1 \mathrm{~m} / \mathrm{s}$.

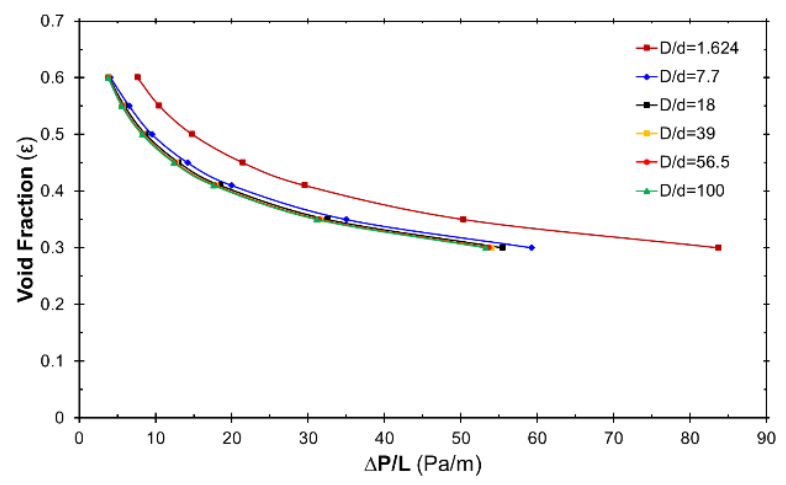

Fig. 3. Pressure drop $(\mathrm{Pa})$ variation depending on the voidage

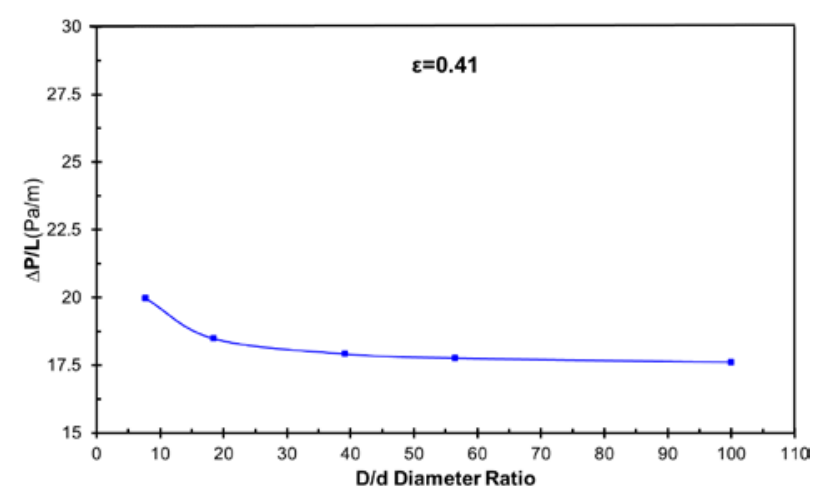

Fig. 4. Pressure drop $(\mathrm{Pa})$ change at constant voidage

In Figure 3, it is seen that the pressure drop values decrease as the voidage increases. However, as the ratio of $\mathrm{D} / \mathrm{d}$ diameter ratio increases with the same voidage, the pressure drop value decreases and converges to a curve. Figure 4 shows the relationship between the pressure drop and $\mathrm{D} / \mathrm{d}$ diameter ratio $(0.41)$ according to the method proposed by [8]. Here, with the increase of $\mathrm{D} / \mathrm{d}$ diameter ratio value, the pressure drop value decreases with time and it converges gradually.

In conclusion, it is stated that the Ergun equation is compatible with the equations developed in the literature, and the voidage values used in the literature are in the range of $0.3-0.5$, and the pressure drop decreases as the voidage increases. In addition, when the relationship between the ratio $\mathrm{D} / \mathrm{d}$ and the voidage is examined, it is seen that the voidage value has not changed after a certain $\mathrm{D} / \mathrm{d}$ diameter ratio value.

As a general approach, the $\mathrm{D} / \mathrm{d}$ ratio $(>15)$ converges to a single curve as the voidage converges to 0.4 (Figure 3 ) and the ratio of pressure drop due to voidage $(>10)$ increases as the $\mathrm{D} / \mathrm{d}$ increases $(\mathrm{D} / \mathrm{d}>15), \Delta \mathrm{P} / \mathrm{L}$ value will be greater than $20 \mathrm{~Pa}$. This value may differ with the number of Re effects.

\subsection{Heat Transfer}

In the case of steady state, the average heat transfer coefficient within a spherical packed bed with heat exchanger (PBHE) can be determined by the proposed calculation approach for packed beds [11]. One of the proposed equations for the calculation of heat transfer in the flow in a packed bed with spheres,

$$
\varepsilon j_{H}=2.06 R e_{D}^{-0.575}
$$

For the heat transfer analyses, cylinder and sphere diameters are varied in a wide range between $0.18-0.2$ $\mathrm{m}$ and $0.0025-0.06$ respectively. The reason for the small size of the heat exchanger is due to the pressure drop. As the cylinder diameter of the PBHE increases, the pressure drop increases and the PBHE efficiency decreases. As mentioned in the pressure drop section, the ratio of the packed bed diameter and the voidage for the sphere intervals are calculated according to the approach (Equation 2) obtained by [8] and the voidage are considered as values ranging from 0.41 to 0.52 .

Colburn $\mathrm{j}$ factor $\left(\mathrm{j}_{\mathrm{H}}\right)$ in Equation 6 can be expressed as a function of Stanton and Prandtl numbers. For this study, Prandtl number has found as 0.7 .

$$
j_{H}=S t \operatorname{Pr}^{2 / 3} \quad 0.6<\operatorname{Pr}<60
$$

Equations 5 and 6 are arranged;

$$
j_{H}=\frac{2.06 R e^{-0.575}}{\varepsilon}=S t P r^{2 / 3}
$$

is obtained.

$$
\operatorname{Pr}=\frac{\mu c_{p}}{k}, \quad S t=\frac{N u}{R e P r}, \quad N u=\frac{\bar{h} D}{k}
$$

Equation 7 can be rearranged by using definitions at equation 8 ,

$$
S t=\frac{2.06 R e^{-0.575} P^{-2 / 3}}{\varepsilon}=\frac{N u}{R e P r}=\frac{\bar{h}}{\rho V c_{p}}
$$

The average heat transfer coefficient for PBHE is:

$$
\bar{h}=\frac{2.06 R e^{-0.575} \mathrm{Pr}^{-2 / 3} \rho V c_{p}}{\varepsilon}
$$

In this study, the average heat transfer coefficient of equation 10 was calculated as $0.0025-0.06 \mathrm{~m}$, cylinder diameter $0.18-0.2 \mathrm{~m}$ and cylinder length $0.1-0.3 \mathrm{~m}$.

The heat transfer coefficient values can also be determined by using the quasi-experimental approaches proposed by [16] together with average heat transfer coefficient calculation as well as by [17].

$$
\begin{gathered}
N u_{l}=0.664 \operatorname{Pr}^{\frac{1}{3}}\left(\frac{R e}{\varepsilon}\right)^{1 / 2} \\
N u_{t}=\frac{0.037\left(\frac{R e}{\varepsilon}\right)^{0.8} \operatorname{Pr}^{-0.1}\left(\operatorname{Pr}^{\frac{2}{3}}-1\right)}{1+2.443\left(\frac{R e}{\varepsilon}\right)^{-0}}
\end{gathered}
$$


where, $\mathrm{Nu}_{1}$ and $\mathrm{Nu}_{\mathrm{t}}$ represent heat transfer on a single sphere in laminar and turbulent flow conditions, respectively. $\mathrm{Nu}$ number $\left(\mathrm{Nu}_{\mathrm{sp}}\right)$ for single sphere using these equations,

$$
N u_{s p}=2+\left(N u_{l}^{2}+N u_{t}^{2}\right)^{1 / 2}
$$

The average heat transfer coefficient $(\overline{N u})$ in the PBHE is dependent on an experimental correction factor $(\mathrm{f}(\varepsilon))$;

$$
\begin{gathered}
f(\varepsilon)=1+1.5(1-\varepsilon) \\
\overline{N u}=f(\varepsilon) N u_{s p}
\end{gathered}
$$

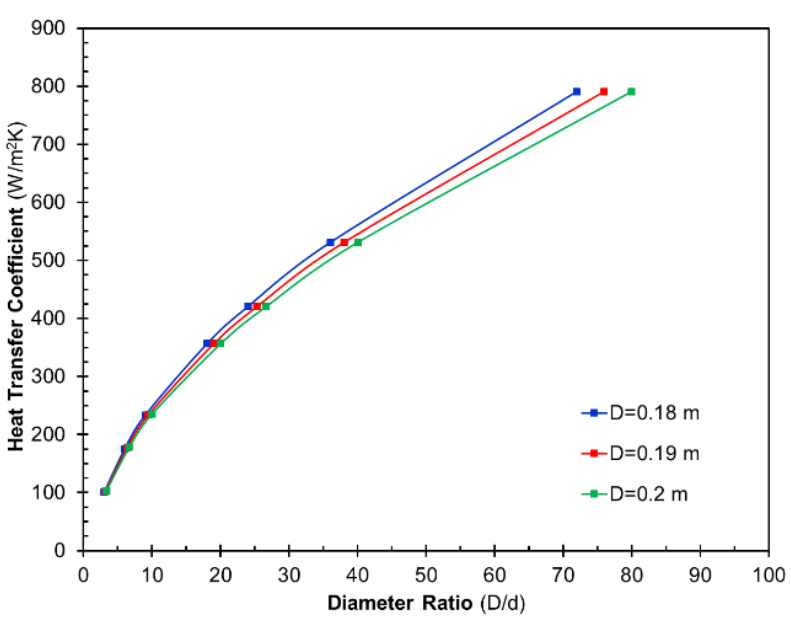

Fig. 5. Heat transfer coefficient values for all diameter ratios

Figure 5 shows the heat transfer coefficient values according to different diameter ratios. Here, it can be seen that the increase of the diameter ratio increases with the increase in the coefficient of heat transfer. The cylinder diameter was used as $0.18 \mathrm{~m}$ for the analyses. This is because a lower pressure drop is obtained at lower diameter ratios.

In this study, one dimensional model which can determine the efficiency for the basic geometric and flow parameters determined during the design of a PBHE is developed. Once the average heat transfer coefficient was determined, it was aimed to determine the efficiency of PBHE by a one-dimensional solution approach. The use of PBHE as a heat recovery ventilation device requires a two-stage process. If we take into account the winter conditions, the hot and polluted air in the heated space in the first stage will be passed through the device and the dimensions, as well as the optimum flowing times. Timedependent heat transfer problems usually result from a sudden change in the thermal environment. Thermal capacitance method is used to solve this problem. The thermal capacitance method is based on the assumption that the temperature in the solid is constant at any point in the time dependent process. This approach ignores the temperature gradients in the solid. The absence of temperature gradients in the solid is in fact impossible.
However, the thermal conductivity coefficient of the solid is sufficiently high, making it possible for us to assume that the temperature distribution in the solid is uniform at every point in the time dependent process. The basic criterion for this assumption is the value of the Biot number (Bi) [11]. Biot number; determines the value of the temperature change in the solid material according to the temperature difference between the surface and the fluid temperature. If the biot number (Bi) is too small, the temperature change in the solid is also very small and the constant temperature assumption is large. There is a general consensus in the literature that the thermal capacitance method can be used for $\mathrm{Bi}<0.1$ values.

In this problem, for a given PBHE, one dimensional mathematical model created and divided into calculation areas along the x-axis as shown in Figure 6, and energy balance equations are created for each cell number according to hot and cold flow states. Here, a mesh is defined as each part we divide the PBHE into equal parts with a constant temperature. These parts represent sphere temperatures in the area they are located. Just as in the finite difference method, when the mesh number increases, the more precise solutions are obtained.

Figure 7 shows the thermal resistances of the generated mathematical model. Here, the heat stored in the spheres in each calculation area in the PBHE together with the air from the hot or cold flow, and the amount of heat transfer transferred to the next calculation area are shown. In the case of hot flow, the temperature of the spheres and the amount of heat stored in the spheres are calculated in equations 16 and 17. The heat transfer from the hot and cold flow to the spheres was investigated separately.

Temperature of one mesh for hot flow:

$$
\frac{\theta}{\theta_{i}}=\frac{T-T_{\infty}}{T_{i}-T_{\infty}}=\exp \left[-\left(\frac{h A_{s}}{\rho V c}\right) t\right]
$$

Here, $\mathrm{T}$ represents the calculation area temperature generated by the effect of the fluid coming from the hot flow state. $T_{\infty}$ is the temperature of the fluid entering into the PBHE from the hot side. V and $\mathrm{c}$ indicate the volume and the specific heat of the spheres.

Stored heat in a mesh:

$$
\dot{Q}_{s p, h}=m_{s p} c_{s p} \frac{d T}{d t}
$$

The $\mathrm{m}_{\mathrm{sp}}$ represents the weight of the spheres. Similar to the hot flow, the temperature and stored heat of each mesh were also calculated in the cold flow.

$$
\begin{gathered}
\frac{\theta}{\theta_{i}}=\frac{T-T_{\infty}}{T_{i}-T_{\infty}}=\exp \left[-\left(\frac{h A_{s}}{\rho V c}\right) t\right] \\
\dot{Q}_{s p, c}=m_{s p} c_{s p} \frac{d T}{d t}
\end{gathered}
$$

For the cold flow, with equations 18 and 19, the temperatures and the stored heat of the spheres can be calculated, respectively. 


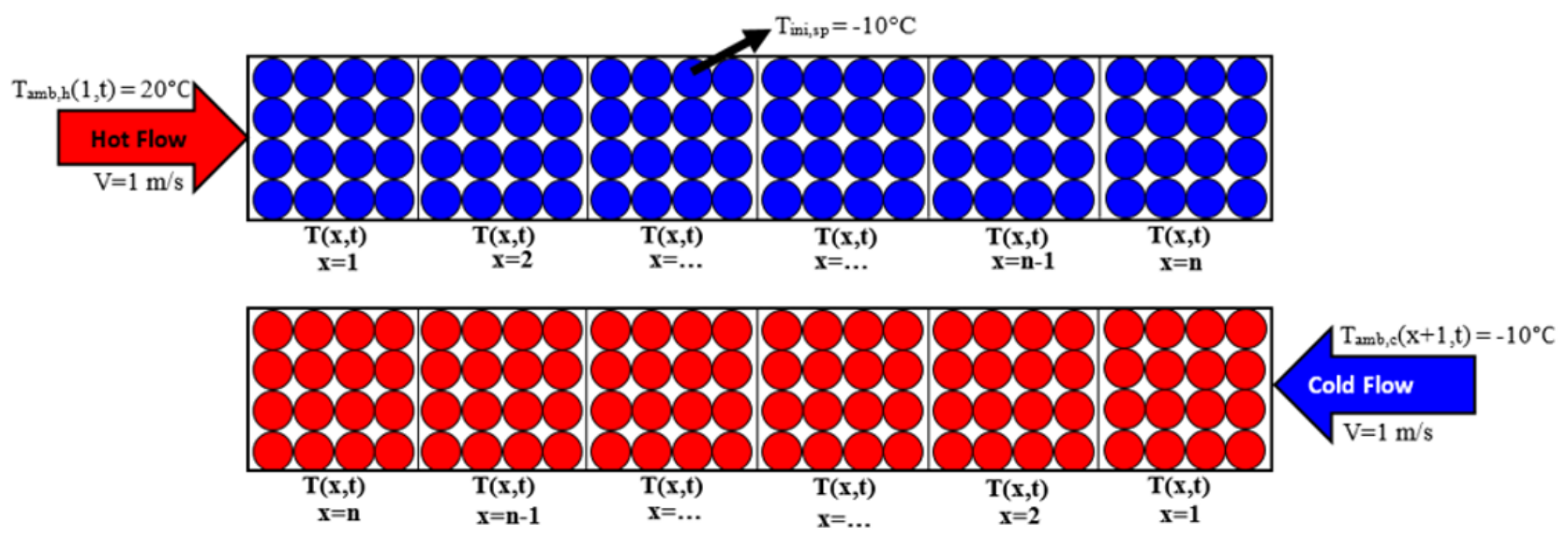

Fig. 6. Mathematical model

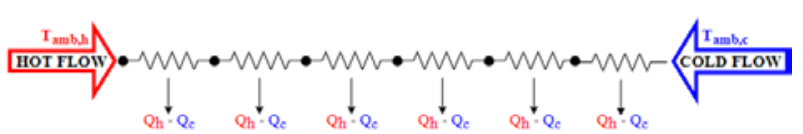

Fig. 7. Heat capacitance model

\section{Results and Discussions}

In Figure 8, the variation of the time distribution of the temperature distribution in the PBHE for the hot flow is shown depending on the location. The first curve represents the temperature values in 5th seconds, the last curve represents the temperature values in 105th seconds and each curve shows the temperature distribution in the PBHE with a difference of 10th seconds. The temperature of the spheres near the PBHE inlet side at 5 seconds is lower than the inlet temperature of the air. Starting from the 15th second, the sphere temperatures are equal to the inlet temperature of the hot air and the spheres reach the limit of their thermal capacity. From the 75 th second to the PBHE exit point, heat is stored in all the spheres in the system and temperature values are higher than $-10{ }^{\circ} \mathrm{C}$ observed at the start time. The longer the hot flow, the higher the temperatures in the PBHE and the more thermal capacity of the sphere.

Due to the fact that the calculation method is one dimensional, temperature distributions in PBHE can be obtained in one dimension. However, the results are presented as two dimensional to provide the temperature distribution visuality. The individual temperature distribution or vertical temperature differences of the spheres are outside the scope of this study. In the actual operating conditions of the system, hot flow and cold flow are applied repeatedly.

The temperature distributions occurring in the PBHE as a result of different flow times for a sequential hot flow and cold flow (one cycle) are shown in Figure 9. Figure 9(a) shows the hot flow and cold flow flowing times of 10 seconds. The temperature of the spheres in the system increases with the air coming from the hot flow. In the case of hot flow (Figure 9.a-1) it can be understood that the heat storage potential of the spheres in the regions different from the highest temperature value (red zone) is

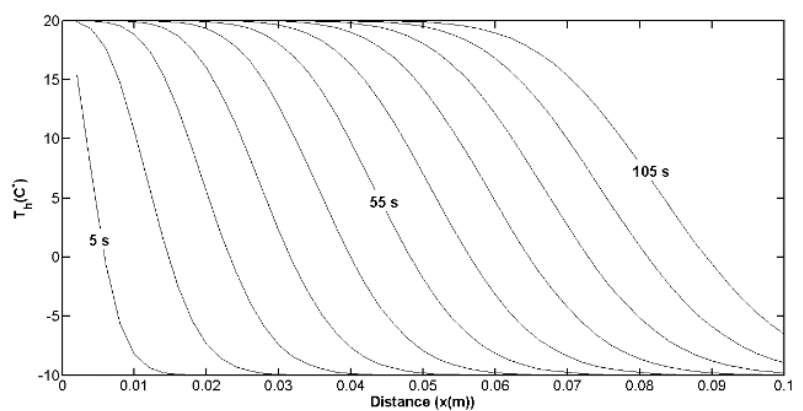

Fig. 8. Temperature values for each location for hot flow

not fully utilized. On the other hand, in the case of cold flow (Figure 9.a-2), it is understood that the heat recovery potential of the heat exchanger is not fully used in areas other than the lowest temperature zone (dark blue). Figure 9 (b), (c), (d) and (e) shows the temperature distributions of the hot and cold flow conditions at different flow times. As the hot flow time increases, the spheres in the PBHE begin to store more heat and the sphere temperatures are equal to the inlet temperature of the hot air. However, as the cold flow time increases, the heat recovery potential of the heat exchanger is increased.

A qualitative evaluation can be made about the efficiency of the system via the temperature distribution graphs given in Figure 9. However, for different designs and working conditions (dimensions, different materials, working times, etc.), it is necessary to determine the efficiency in order to quantify the performance. In heat recovery systems, efficiency is defined as the ratio of recovered heat to the highest amount of heat that can be recovered and expressed in Equation 20.

$$
\eta=\frac{T_{c}-T_{a m b, c}}{T_{a m b, h}-T_{a m b, c}} x 100
$$

Figure 10 shows the change in efficiency values obtained at different hot and cold flow times according to the number of cycles. In hot and cold flow, the efficiency of the PBHE increases as the flow time decreases. However, as the number of cycles increases, the PBHE efficiency increases first and after a period of time, stable periodic working conditions are formed and the efficiency is fixed.

\footnotetext{
* Corresponding author: alper.m.genc@gmail.com
} 
As the hot and cold flow time increases, the system becomes stable at a lower cycle number. For lower flow

Hot Side

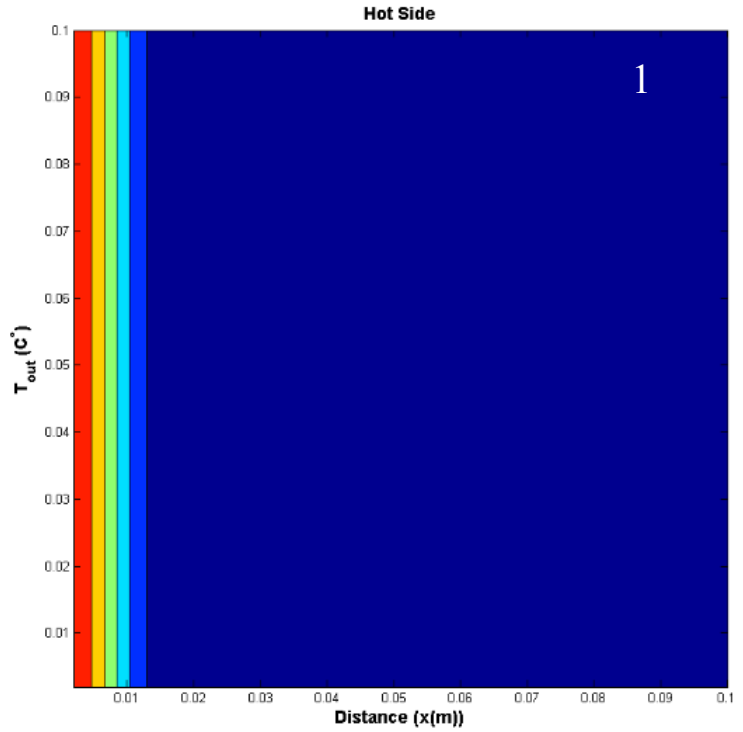

(a) $t=10 s$

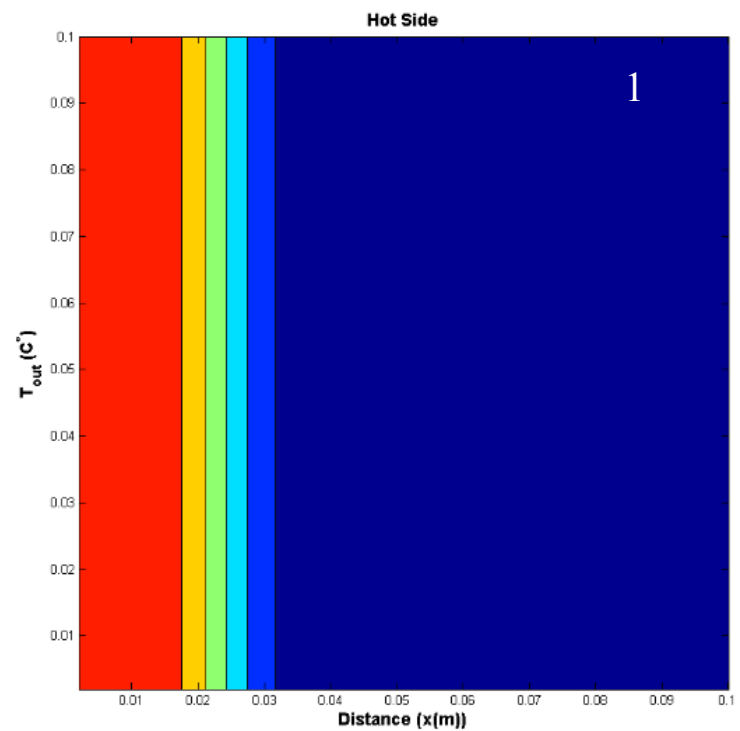

(b) $\mathrm{t}=30 \mathrm{~s}$

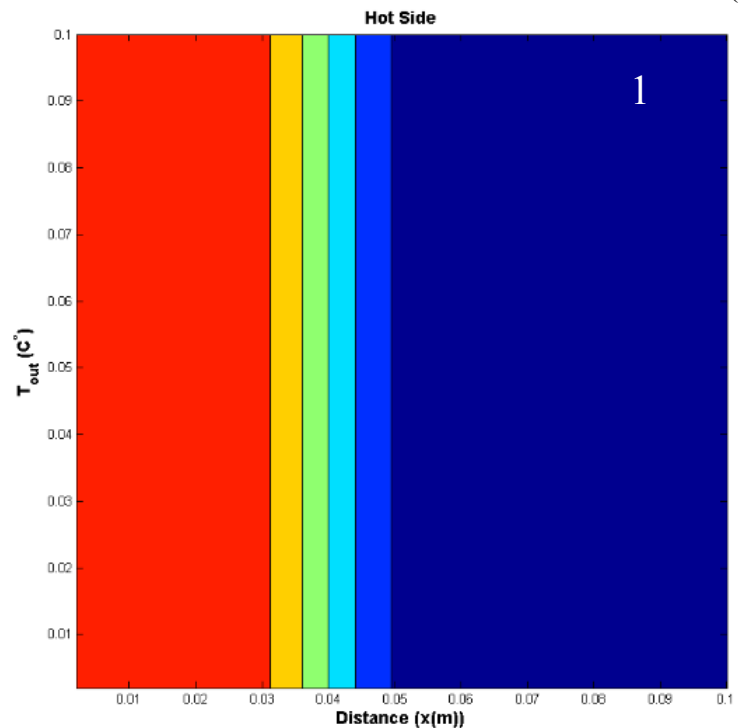

times, a higher number of cycles is required for the system to reach steady state.

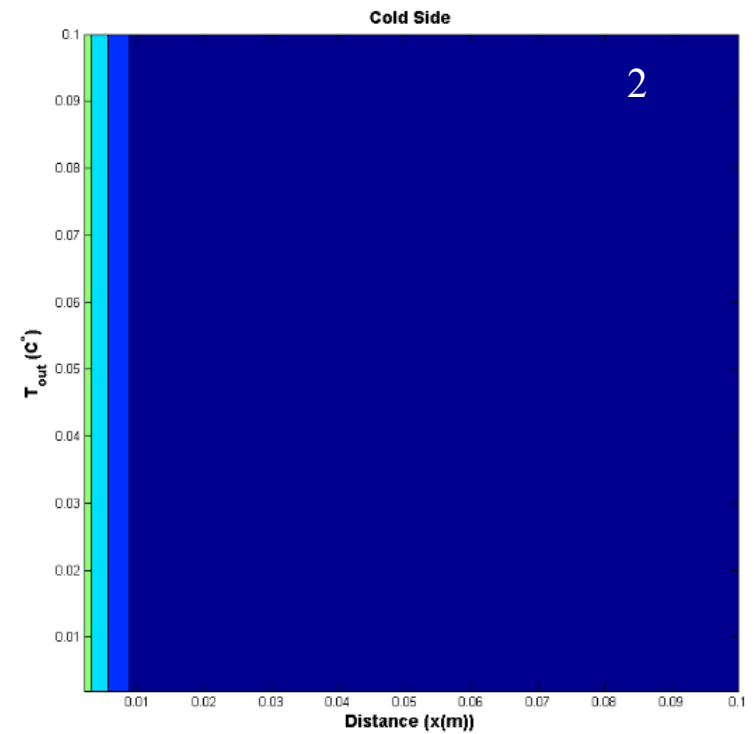

Cold Side

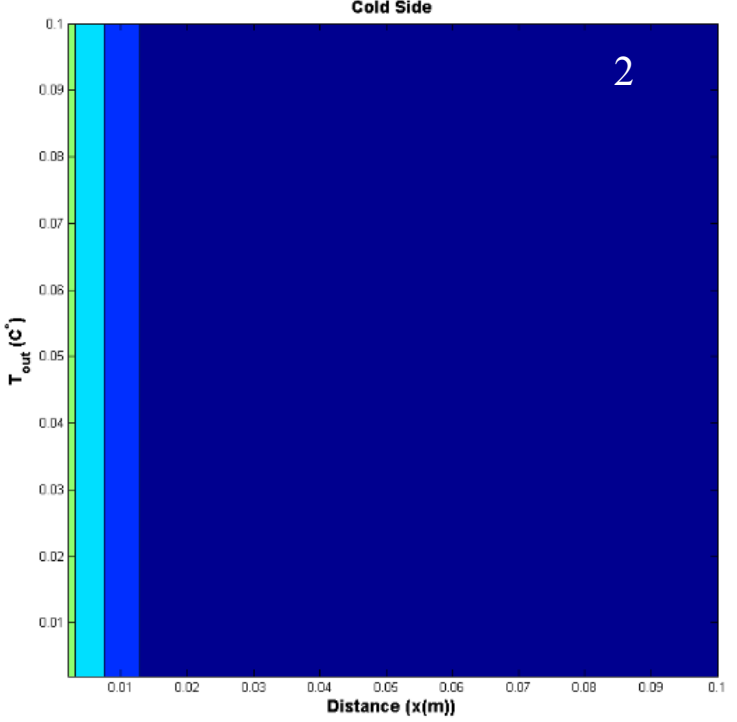

Cold Side

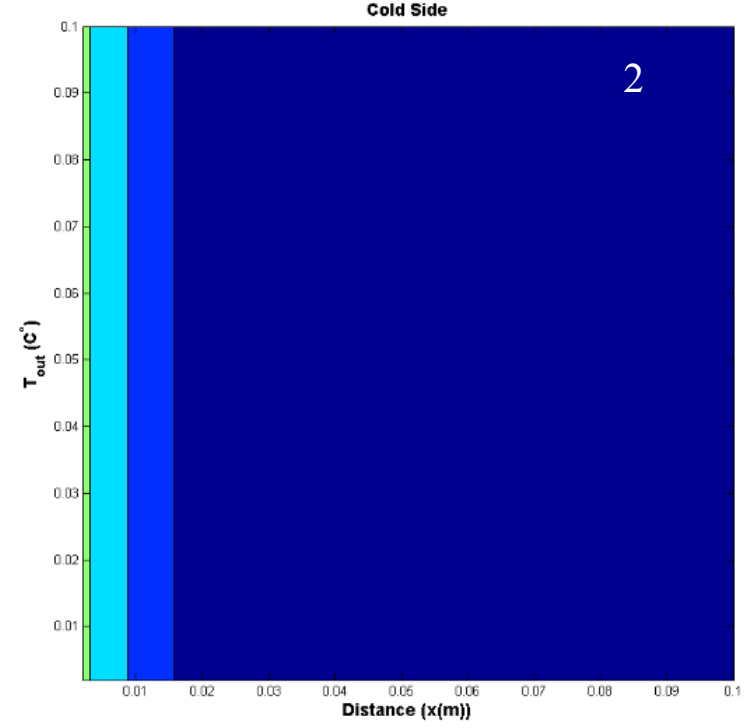

(c) $t=50 s$ 

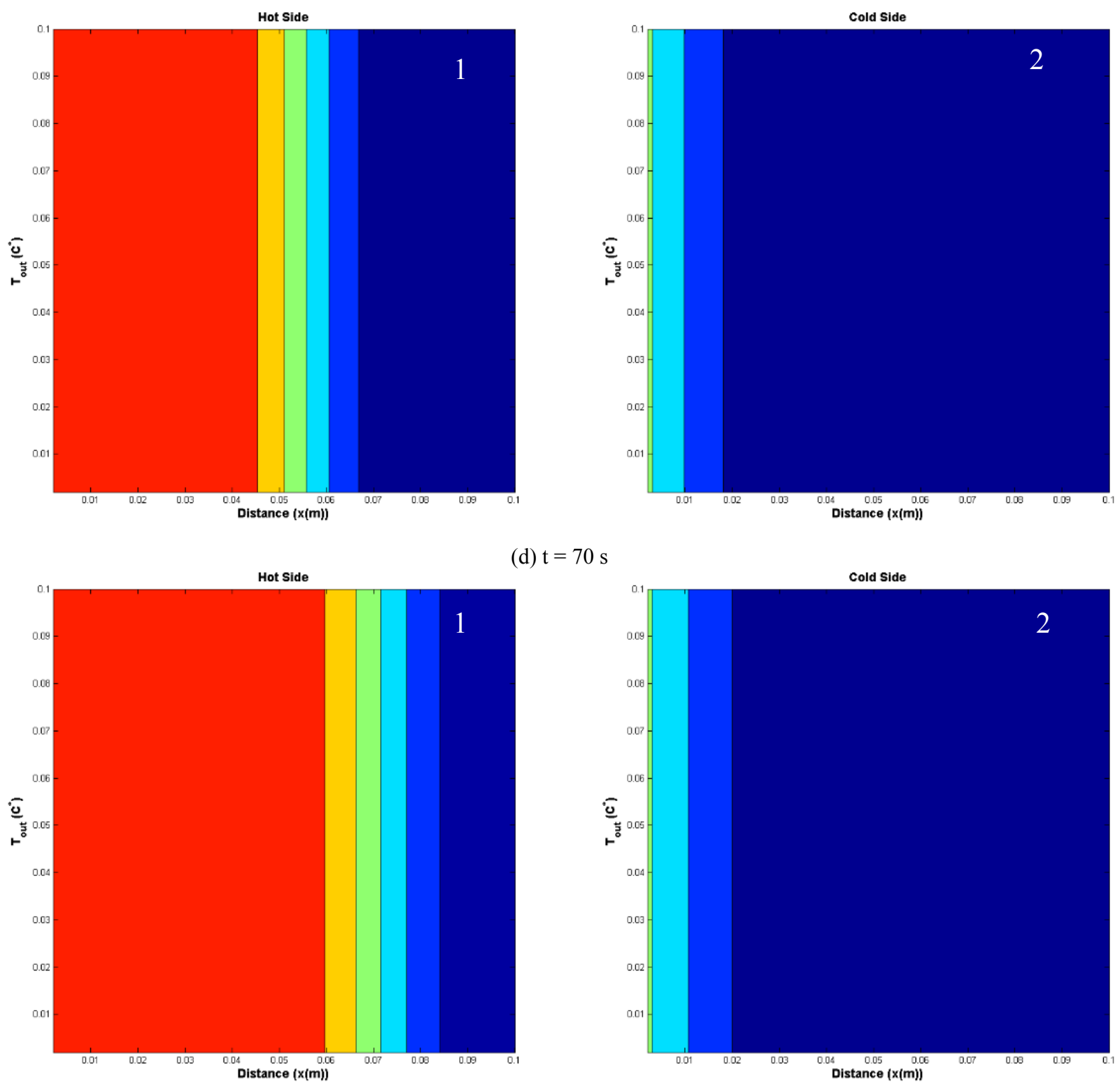

d) $\mathrm{t}=70 \mathrm{~s}$

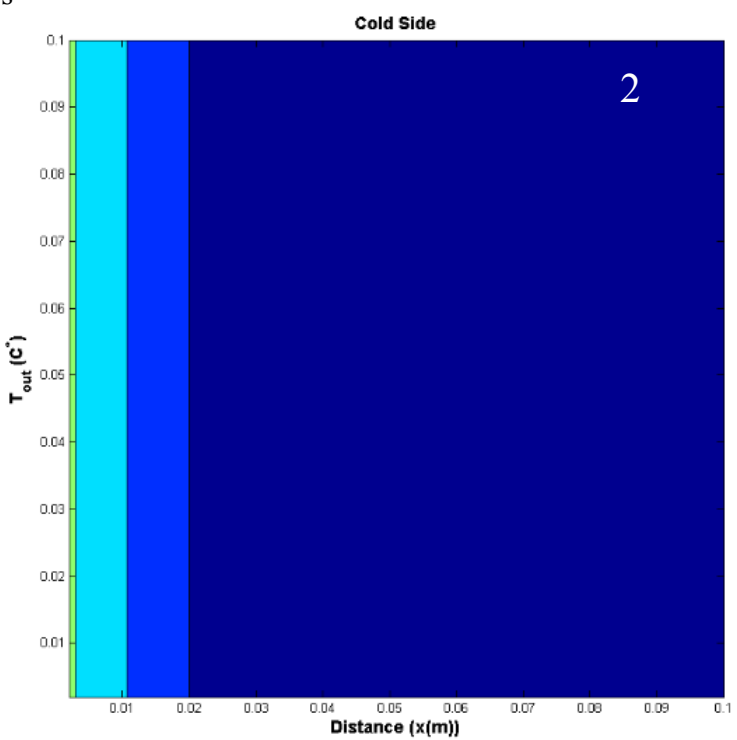

(e) $t=90 s$

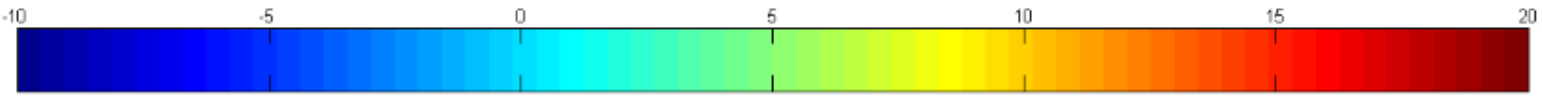

Figure 9. Time-dependent temperature distribution of the system for one cycle

However, considering the total operating times, it is observed that the system becomes more stable and stable when the blowing time becomes shorter.

The highest efficiency was obtained at about $93 \%$, hot and cold flow time was 5 seconds. In this case, the stable operating efficiency is expected to be slightly higher as the system does not yet reach stable working conditions. The lowest efficiency was obtained in approximately $76 \%$ when the hot and cold flow time was 90 seconds.

\section{Conclusion}

In this study, transient numerical analyses of spherical packed beds for periodic flow investigated. Bed diameters

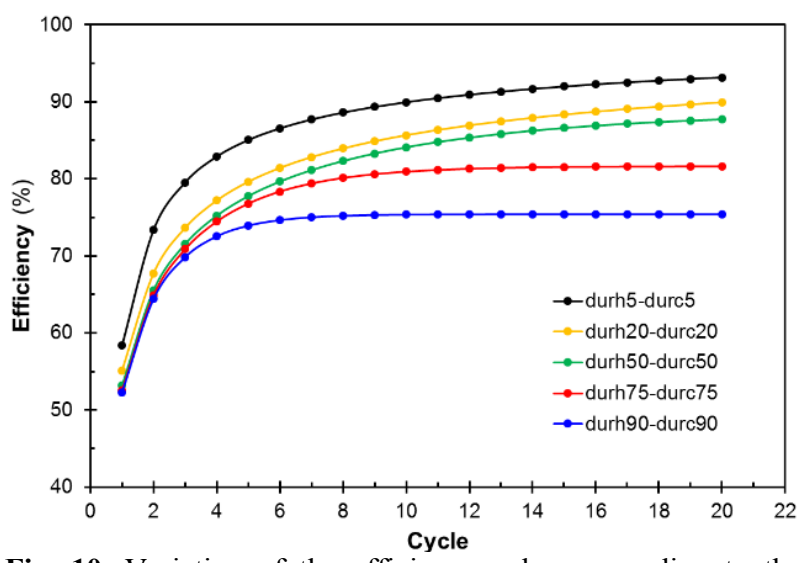

Fig. 10. Variation of the efficiency values according to the number of cycles 
were studied ranging between $0.18-0.2 \mathrm{~m}$. Spherical diameters were chosen in a wide range of $0.0025-0.06 \mathrm{~m}$. Various flow time and number of cycles were investigated for the hot and cold flow to understand the spherical bed performance for HRV applications.

The main conclusions are presented as follows:

- As the number of cycles increases, the PBHE efficiency increases first and after a period of time, stable periodic working conditions are formed and the efficiency is fixed.

- As the hot and cold flow time increases, the system becomes stable at a lower cycle number. For lower flow times, a higher number of cycles is required for the system to reach steady state.

- Considering the total operating times, it is observed that the system becomes more stable and stable when the blowing time becomes shorter.

- The highest efficiency was obtained at about $93 \%$, hot and cold flow time was 5 seconds.

\section{References}

1. Martin, H., Low Peclet number particle-to-fluid heat and mass transfer in packed beds. Chemical Engineering Science, 33(7), 913-919 (1978)

2. Nemec, D., \& Levec, J. Flow through packed bed reactors: 1. Single-phase flow. Chemical Engineering Science, 60(24), 6947-6957 (2005)

3. Yu, J., Zhang, M., Fan, W., Zhou, Y., Zhao, G.: Study on performance of the ball packed-bed regenerator: experiments and simulation. Appl. Therm. Eng. 22, 641651 (2002)

4. Toghraie, D., Afrand, M., Zadeh, A. D., \& Akbari, H. A., Numerical investigation on the flow and heat transfer of a multi-lobe particle and equivalent spherical particles in a packed bed with considering the wall effects. International Journal of Mechanical Sciences, 138, 350367 (2018)

5. Guo, Z., Sun, Z., Zhang, N., Ding, M., \& Cao, X., Radial porosity peak at the centerline of packed beds with small tube to particle diameter ratios. Powder Technology, 319, 445-451 (2017)

6. Allen, K. G., Von Backström, T. W., \& Kröger, D. G., Packed bed pressure drop dependence on particle shape, size distribution, packing arrangement and roughness. Powder technology, 246, 590-600 (2013)

7. Jeschar, R., Druckverlust in MehrkornschuK ttungen aus Kugeln. Archiv Eisenhu (ttenwesen, 35, 91) 108 (1964)
8. De Klerk, A. Voidage variation in packed beds at small column to particle diameter ratio. AIChE journal, 49(8), 2022-2029 (2003).

9. Choi, Y. S., Kim, S. J., \& Kim, D., A semi-empirical correlation for pressure drop in packed beds of spherical particles. Transport in porous media, 75(2), 133-149 (2008)

10. Eisfeld, B., \& Schnitzlein, K., The influence of confining walls on the pressure drop in packed beds. Chemical Engineering Science, 56(14), 4321-4329 (2001)

11. Bergman, T. L., Incropera, F. P., DeWitt, D. P., \& Lavine, A. S., Fundamentals of heat and mass transfer. John Wiley \& Sons (2011)

12. Ergun, S., Fluid flow through packed columns. Chem. Eng. Prog. 48, 89-94 (1952)

13. Mehta, D., Hawley,M.C.,Wall effect in packed columns. Ind. Eng. Chem. Proc. Des. Dev. 8, 280-282 (1969)

14. Augier, F., Idoux, F., \& Delenne, J. Y., Numerical simulations of transfer and transport properties inside packed beds of spherical particles. Chemical Engineering Science, 65(3), 1055-1064 (2010)

15. Guardo, A., Coussirat, M., Larrayoz, M. A., Recasens, F., \& Egusquiza, E. Influence of the turbulence model in CFD modeling of wall-to-fluid heat transfer in packed beds. Chemical Engineering Science, 60(6), 1733-1742 (2005)

16. Achenbach, E., Heat and flow characteristics of packed beds. Experimental thermal and fluid science, 10(1), 17-27 (1995)

17. Gnielinski, V., Gleichungen zur Berechnung des W irme- und Stoffaustausches in durchstr6mten ruhenden Kugelschiittungen bei mittleren und grossen Pecletzahlen, Verfahrenstechnik 12(6), 63-366 (1978) 